\title{
VENTILAÇÃO MECÂNICA: EVIDÊNCIAS PARA O CUIDADO DE ENFERMAGEM
}

\author{
Ventilación mecánica: evidencia para el cuidado en enfermería \\ Mechanic ventilation: evidence for nursing care
}

Yarla Cristine Santos Jales Rodrigues ${ }^{1}$

Maria do Carmo de Oliveira Citó ${ }^{4}$
Rita Mônica Borges Studart²

Elizabeth Mesquita Melo ${ }^{5}$
Ítalo Rigober to Cavalcante Andrade ${ }^{3}$

Islene Victor Barbosa ${ }^{6}$

\section{RESUMO}

Este estudo objetivou avaliar o conhecimento dos enfermeiros sobre ventilação mecânica nas unidades de terapia intensiva (UTIs) de um hospital de referência em Fortaleza. Trata-se de um estudo transversal, quantitativo. A amostra foi constituída por 43 enfermeiros, com aplicação de questionário. A coleta de dados ocorreu em fevereiro e março de 2011. Os dados foram transcritos e tabulados no programa Excel, organizados em tabelas, interpretados e fundamentados com base na literatura pertinente. A maioria dos sujeitos é do sexo feminino $(88,4 \%)$ e não especialistas em UTI (79,0\%); o tempo de atuação em UTI prevaleceu de zero a cinco anos (48,8\%). A avaliação da ciclagem do ventilador mecânico a volume não foi satisfatória, $65,1 \%$. A participação do enfermeiro é mínima na definição de parâmetros, extubação, desmame e aspiração. Conclui-se que o enfermeiro necessita ampliar o seu conhecimento em ventilação mecânica, e os programas de treinamentos nesta área devem ser incentivados pelas instituições de trabalho, no intuito de qualificar a assistência de enfermagem a estes pacientes.

Palavras-chave: Enfermagem. Ventilação mecânica. Unidade de terapia intensiva.

\section{Abstract}

To assess nurses' knowledge related to mechanical ventilation in intensive care units (ICUs) of a referral hospital in Fortaleza. This is a Cross-sectional and quantitative study. Sample was made by nurses in ICUs on a total of 43 nurses, with a questionnaire. Data collection was conducted between February and March 2011. The analysis, data were transcribed and tabulated in Excel program, organized in tables, interpreted and justified based on the literature pertinent. Majority female (88.4\%), time in the ICU prevailed from zero to five years $(48.8 \%)$, most of the nurses are not experts in the ICU (79.0\%). The evaluation of the cycling of the mechanic ventilation at volume was not satisfactory, $65.1 \%$. It was noted that the participation of nurses is minimal in defining parameters, in participation of extubation, weaning and aspiration. It was concluded that nurses must expand their knowledge and implement nursing care to patients in mechanical ventilation. The training programs on mechanic ventilation for nurses should be encouraged by working institutions in order to qualify the nursing care to patients in need of ventilatory support.

Keywords: Nursing. Mechanical ventilation. Intensive care unit.
Evaluar los conocimientos de los enfermeros sobre la ventilación mecánica en unidades de cuidados intensivos (UCl) de un hospital de referencia en Fortaleza (CE). Estudio trasversal, cuantitativo. Muestra constituida por 43 enfermeras, con aplicación de una encuesta. Los datos fueron recolectados entre febrero y marzo de 2011 y transcritos y tabulados en el programa Excel, organizados en tablas, interpretado y justificado sobre la base de la documentación pertinente. Resultados: Mayoría femenina $(88,4 \%)$; Tiempo en la UCl entre zero y cinco años (48,8\%); Mayoría no son expertos en la UCI (79,0\%); La evaluación de la ventilación mecánica por volumen no era satisfactoria $(65,1 \%)$. La participación de los enfermeros es mínima en la definición de los parámetros, extubación, desmame y aspiración. Se concluye que los enfermeros necesitan ampliar su conocimiento sobre la ventilación mecánica y los programas de capacitación en esta área deben ser fomentados por las instituciones que trabajan para cualificar la asistencia en enfermería a estos pacientes.

Palabras clave: Enfermería. Ventilación mecánica. Unidad de cuidados intensivos.

\footnotetext{
${ }^{1}$ Enfermeira, especialista em Unidade de Terapia Intensiva. Fortaleza-CE. Brasil. E-mail: yarlinha_cris@hotmail.com; ²Enfermeira, mestre em Enfermagem pela Universidade Federal do Ceará. Professora da Universidade de Fortaleza. Centro de Ciências da Saúde. Fortaleza-CE. Brasil. E-mail: monicastudart@hotmail.com; ${ }^{3}$ Enfermeiro, especialista em Unidade de Terapia Intensiva. Professor da Universidade de Fortaleza. Centro de Ciências da Saúde. Fortaleza-CE. Brasil. E-mail: italorigo@unifor.br; “ ${ }^{2}$ nfermeira, mestre em Farmacologia pela Universidade Federal do Ceará. Fortaleza-CE. Brasil. Email: cacacit@yahoo.com.br; ${ }^{5}$ Enfermeira, doutora em Enfermagem pela Universidade Federal do Ceará. Professora da Universidade de Fortaleza. Centro de Ciências da Saúde. Fortaleza-CE. Brasil. E-mail: elizjornet@yahoo.com.br; ${ }^{6}$ Enfermeira, doutora em Enfermagem pela Universidade Federal do Ceará. Professora da Universidade de Fortaleza. Centro de Ciências da Saúde. Fortaleza-CE. Brasil. E-mail: islene1@hotmail.com
} 


\section{INTRODUÇÃO}

Há muitos anos, a ventilação artificial vem sendo desafiada, na tentativa de substituir da melhor forma a função respiratória. Desde os primeiros experimentos por Vesalius e Hooke com o tórax aberto de um animal, nos quais foi demonstrado que a vida poderia ser preservada a partir da insuflação dos pulmões com um balão de ar, passando pelos primeiros ventiladores mecânicos por pressão negativa, nos quais os pacientes ficavam aprisionados no interior de câmaras fechadas para manter a ventilação e oxigenação de forma artificial, até chegar aos dias atuais, o suporte ventilatório é assunto muito estudado e discutido ${ }^{1}$

Os centros de pólio trouxeram novas tecnologias de ventilação mecânica a partir da década de 1950, em meio a uma epidemia de poliomielite, com o uso de ventiladores por pressão positiva com os quais os pulmões dos pacientes contaminados eram ventilados manualmente por voluntários. Ao longo dos anos, os ventiladores foram evoluindo sistematicamente, possibilitando novas intervenções e monitorizações ${ }^{2}$

A ventilação mecânica (VM) é um dos suportes à vida de grande importância em UTI e constitui um dos recursos mais utilizados nessas unidades, consistindo no emprego de uma máquina que substitui, total ou parcialmente, a atividade ventilatória do paciente, com o objetivo de restabelecer o balanço entre a oferta e a demanda de oxigênio e atenuar a carga de trabalho respiratório de pacientes com insuficiência respiratória.

Com a criação das UTIs na década de 1960, a utilização da VM cresceu bastante e tornou-se rotina nessas unidades para a recuperação da maioria dos pacientes críticos. Apesar do desenvolvimento tecnológico, o prognóstico desses pacientes permanecia reservado, o que se tornou preocupante para os profissionais que atuavam nessas unidades, pois a taxa de mortalidade dos pacientes com insuficiência respiratória ainda era muito alta. ${ }^{3}$

A partir de 1985 percebeu-se que a VM também poderia trazer malefícios e o conceito de lesão induzida pela ventilação artificial recebeu uma atenção especial, pois logo foi comprovado que a VM inadequada era capaz de lesar as microestruturas pulmonares e ser deletéria ou até fatal para o paciente. ${ }^{4}$

Tendo em vista o elevado número de pacientes internados em UTI que estão em uso de VM, é de suma importância que os enfermeiros estejam capacitados a prestar cuidados inerentes à monitorização dos parâmetros ventilatórios e dos alarmes, à mobilização, à remoção de secreções, ao aquecimento e à umidificação dos gases inalados, bem como ao controle das condições hemodinâmicas do paciente, visando a minimizar os efeitos adversos. ${ }^{5}$

A atenção aos pacientes sob VM torna-se responsabilidade dos enfermeiros, pois a evolução positiva deles depende de cuidados contínuos, capazes de promover a identificação de problemas que atinjam diretamente suas necessidades. Para uma prestação de cuidado de qualidade é necessário que os enfermeiros tenham uma ampla compreensão dos princípios da VM, além de reconhecer a tolerância fisiológica específica de cada paciente. ${ }^{6}$

0 Conselho Federal de Enfermagem (Cofen) foi enfático ao determinar, mediante a Lei $n .7 .498 / 86$, que compete ao enfermeiro a execução de cuidados diretos de enfermagem a pacientes graves com risco de morte, além de cuidados de enfermagem de maior complexidade técnica, que exijam conhecimentos de base científica e capacidade de tomar decisões. ${ }^{7}$ E trabalhar em um ambiente de terapia intensiva requer capacitação profissional, investimento intensivo no cuidado de pacientes instáveis, uso de tecnologias variadas e convívio com morte, estresse e conflitos. ${ }^{8}$

0 enfermeiro atua fundamentalmente em UTIs desde que estas se especializaram no atendimento a pacientes graves; porém, observa-se também que os enfermeiros, atualmente, em determinadas instituições, estão ficando cada vez mais distantes do suporte ventilatório, talvez pelas inúmeras atribuiç̃oes que lhes são destituídas, ou por haver outra categoria profissional fazendo esse tipo de assistência, bem como pela deficiência de seu conhecimento.

Para fornecer um suporte ventilatório mecânico para um paciente, o enfermeiro deve saber manusear os diversos ventiladores. Os fisioterapeutas respiratórios compartilham essa responsabilidade de controlar o ventilador em algumas instituições, mas, mesmo nessa situação, o enfermeiro deve estar totalmente ciente das implicações para o paciente do modo e nível do suporte mecânico. ${ }^{9}$

Para obtenção de um resultado positivo aos pacientes submetidos a suporte ventilatório, é necessária a compreensão dos princípios da VM, assim como das necessidades de cuidados do paciente, bem como da comunicação aberta entre os membros da equipe de saúde sobre as metas da terapia, planos para o desmame e tolerância do paciente em relação às alterações nos parâmetros ventilatórios. ${ }^{6}$

0 enfermeiro, ao monitorar o ventilador, deve observar: o tipo de ventilador; as modalidades de controle; os parâmetros de volume corrente e frequência respiratória; os parâmetros de fração de inspiração de oxigênio ( $\left.\mathrm{FiO}_{2}\right)$; a pressão inspiratória alcançada e limite de pressão; a relação inspiração/expiração; o volume minuto; os parâmetros de suspiro, quando aplicáveis; a verificação da existência de água no circuito e nas dobras ou a desconexão das traqueias; a umidificação e a temperatura; os alarmes, que devem estar ligados e funcionando adequadamente; e os níveis da pressão positiva no final da expiração (PEEP) e/ou suporte de pressão, quando aplicável. ${ }^{6}$

0 estudo é importante, visto que promoverá considerações relevantes para o enfermeiro ao oferecer subsídios aos que assistem pacientes em uso de ventilação mecânica, para que estes prestem uma assistência sistemática e holística pautada em cuidados com embasamento técnico e 
científico, pois o conhecimento aprofundado se faz necessário para que possam garantir uma assistência eficaz, contribuindo para a melhora do estado clínico do paciente.

Outro ponto que merece destaque é a observação de que atualmente especialistas de outras áreas desempenham funções anteriormente exercidas por enfermeiros. Essa afirmativa induz a uma profunda reflexão sobre mudanças de paradigma, pois o enfermeiro continua sendo holisticamente responsável pelo bem-estar do paciente e não pode estar fora do contexto do suporte ventilatório.

Diante dessa temática, o estudo buscou avaliar o conhecimento do enfermeiro referente à ventilação mecânica nas unidades de terapia intensiva de um hospital de referência.

\section{MÉTODO}

Estudo transversal com abordagem quantitativa. Realizado em três UTIs de um hospital público terciário localizado em Fortaleza-CE, centro de referência no Estado.

Os critérios de exclusão foram: ter menos de seis meses de experiência em UTI, ser enfermeiro afastado (licença e/ou férias) ou enfermeiro coordenador que não prestava assistência direta ao paciente. Dos 54 enfermeiros assistenciais, 43 $(79,6 \%)$ compuseram a amostra.
A coleta de dados ocorreu entre fevereiro e março de 2011, a partir de um questionário estruturado sobre o tema em questão. Para a análise, os dados foram transcritos no programa Microsoft Excel, versão Windows XP Profissional, e organizados em tabelas, as quais foram interpretadas e fundamentadas com base na literatura pertinente.

0 estudo foi realizado tendo por base a Resolução $n$. 196/96, do Conselho Nacional de Saúde. ${ }^{10}$ No intuito de atender aos aspectos éticos, o projeto foi aprovado pelo Comitê de Ética em Pesquisa do hospital, sob o Protocolo n. 396.802 .

\section{RESULTADOS E DISCUSSÃO}

A ventilação mecânica é, sem dúvida, um aspecto importante a ser considerado na terapia intensiva, pois envolve muitas variáveis e necessita ter uma interação interdisciplinar, por sua complexidade tecnológica. É de suma importância que o enfermeiro esteja inserido com competência e habilidades nesse contexto para prestar uma assistência segura ao paciente crítico.

Os enfermeiros participantes do estudo foram caracterizados em relação às variáveis sexo, tempo de graduação, atuação e especialização em UTI, de acordo com a Tabela 1.

TABELA1 - Distribuição dos enfermeiros, segundo o sexo, tempo de graduação, atuação e especialização em UTI. Fortaleza 2011.

\begin{tabular}{|c|c|c|}
\hline SEXO & $\mathbf{N}$ & $\%$ \\
\hline Masculino & 5 & 11,6 \\
\hline Feminino & 38 & 88,4 \\
\hline Total & 43 & 100 \\
\hline TEMPO DE GRADUAÇĀ0 & $\mathbf{N}$ & $\%$ \\
\hline Até 5 anos & 21 & 48,8 \\
\hline De 6 a 10 anos & 9 & 20,9 \\
\hline De 11 a 15 anos & 4 & 9,3 \\
\hline De 16 a 20 anos & 2 & 4,7 \\
\hline+ De 20 anos & 7 & 16,3 \\
\hline Total & 43 & 100,0 \\
\hline TEMPO DE ATUAÇĀO EM UTI & $\mathbf{N}$ & $\%$ \\
\hline Até 5 anos & 25 & 58,1 \\
\hline De 6 a 10 anos & 9 & 21,0 \\
\hline De 11 a 15 anos & 5 & 11,6 \\
\hline De 16 a 20 anos & 3 & 7,0 \\
\hline+ De 20 anos & 1 & 2,3 \\
\hline Total & 43 & 100,0 \\
\hline ESPECIALISTA EM UTI & $\mathbf{N}$ & $\%$ \\
\hline SIM & 9 & 21,0 \\
\hline NÄO & 34 & 79,0 \\
\hline Total & 43 & 100,0 \\
\hline
\end{tabular}

De acordo com os dados, percebe-se que a maioria dos enfermeiros era do sexo feminino, $38(88,4 \%)$. Esse fato demonstra que a enfermagem ainda é uma profissão na qual é predominante o número de mulheres. Entretanto, cuidar é uma qualidade inerente aos seres humanos, e não uma qualidade apenas feminina. ${ }^{11}$

Esse dado coincide com o perfil geral de enfermeiros no Brasil, onde há predomínio do sexo feminino. Os indicadores e dados básicos do Brasil de 2004 corroboram a descrição desse perfil, pois aproximadamente $92 \%$ do total de enfermeiros eram do sexo feminino. ${ }^{12}$

Analisando o tempo de graduação, percebeu-se um destaque nos extremos, com uma predominância de $21(48,8 \%)$ enfermeiros com poucos anos de formação (até cinco anos) perfazendo a maioria dos profissionais atuantes e de uma minoria, $2(4,7 \%)$, entre 16 a 20 anos de formação. Foi predominante o pouco tempo de formação dos enfermeiros, indicando que a instituição contrata profissionais com pouca 
experiência e que estes são treinados no serviço. Isso coincide com a realidade de outras UTIs, como no interior de São Paulo, que possui um quadro de $42,8 \%$ dos enfermeiros com experiência entre 1 e 4 anose $38 \%$ com experiência de 5 a 8 anos. ${ }^{13}$

Em relação ao tempo de atuação em UTI foi constatado que $25(58,1 \%)$ tinham até 5 anos de atuação com pacientes críticos e apenas 1 enfermeiro (2,3\%) tinha mais de 20 anos trabalhando em UTI, dados relativamente similares aos do tempo deformação.

Embora o pouco tempo de formação denote pouca experiência prática, esses profissionais possuem conhecimento teórico proporcionado pela grade curricular do curso de graduação, que possibilita a atuação em UTI. Foi percebido que os enfermeiros que participaram do estudo estão sempre em busca de aprimoramento e especialização, talvez isso seja a justificativa da instituição para contratar esses profissionais. A convivência com os profissionais mais experientes facilita a troca de conhecimento, oferecendo maior segurança Assim, os enfermeiros necessitam ser treinados para pôr em prática seus conhecimentos, e não simplesmente assumir escalas sem dispor desse treinamento.

Em relação à titulação, apenas nove $(21,0 \%)$ tinham especialização em UTI e a maioria, 34 (79,0\%), não apresentava essa capacitação. Percebe-se, portanto, a existência de muitos enfermeiros trabalhando em terapia intensiva sem ser especialistas. Esses enfermeiros buscam os cursos de aperfeiçoamento e especialização em áreas afins.

Uma considerável redução do número de enfermeiros com mais de 20 anos atuando em UTI pode estar relacionada ao fato de que esses profissionais, quando atingem muito tempo de formação, são absorvidos por outros setores; foi observado que eles almejam cargos administrativos, buscam a área de ensino ou até mesmo desistem da profissão. ${ }^{13}$ A vivência diária naUTl e o estresse podem levar os profissionais a mudar sua área de atuação, desejar sair dessas unidades para desempenhar atividades administrativas e de ensino. Essa é uma justificativa empírica para justificar o grande número de profissionais com pouco tempo de atuação e a quantidade tão pequena, quase inexistente, de profissionais com mais de 15 anos de atuação em UTI. ${ }^{11}$

TABELA 2- Distribuição da programação dos parâmetros da ventilação mecânica segundo o conhecimento dos enfermeiros da UTI. Fortaleza, 2011.

\begin{tabular}{lll}
\hline $\begin{array}{l}\text { Resposta esperada relacionada à ciclagem do VM (cidado a } \\
\text { volume). }\end{array}$ & $\mathbf{N}$ & \% \\
\hline Sim & 15 & 34,9 \\
Não & 17 & 39,5 \\
Em branco & 11 & 25,6 \\
\hline Total & 43 & 100.0 \\
\hline Definiçao de Parâmetros & $\mathbf{N}$ & \% \\
Enfermeiro & 2 & 4,7 \\
Fisioterapeuta & 41 & 95,3 \\
\hline Total & 43 & 100.0 \\
\hline
\end{tabular}

De acordo com a Tabela 2, percebe-se que apenas $15(34,9 \%)$ responderam corretamente acerca dos ventiladores por pressão positiva ciclados em volume. A maioria respondeu incorretamente, 17 (39,5\%), e 11 $(25,6 \%)$ deixaram em branco por não saber a resposta. Ao somar as respostas erradas com as não respondidas, temse uma maioria de enfermeiros, $65,1 \%$ (28), que trabalhavam com pacientes em VM mas não sabiam o básico dos parâmetros do ventilador. As respostas foram consideradas sim, ou seja, acerto, quando o enfermeiro respondeu corretamente as três perguntas do instrumento de coleta de dados.

Quanto às definições dos parâmetros do ventilador mecânico, notou-se uma participação significativa do fisioterapeuta, 41 (95,3\%), e uma participação mínima do enfermeiro, apenas $2(4,7 \%)$.

Analisando a variável modalidade de um ventilador mecânico ciclado em volume, foram colocadas três perguntas que mostravam os parâmetros programáveis e, a partir desses dados, o pesquisado responderia qual o tipo de modalidade, se assistida, controlada, ventilação mandatória intermitente sincronizada ou pressão positiva contínua nas vias aéreas. Era considerada resposta correta, ou seja, o sim, quando as três respostas eram verdadeiras e não, para todas as respostas com pelo menos um item errado. Em branco referiu-se a todas as perguntas sem resposta. Grande parte dos enfermeiros do estudo respondeu de modo incorreto ou não soube relacionar.

Espera-se dos profissionais de enfermagem que atuam nessas UTIs conhecimento cientifico apurado, que acompanhem as mudanças tecnológicas e que sejam altamente especializados. $^{2}$

$\mathrm{Na}$ Tabela 3 encontram-se as modalidades do ventilador mecânico separadamente e constata-se um número de acertos bem maior, em que a maioria, 25 (58,1\%), soube relacionar os parâmetros com a modalidade assistida/controlada ( $A / C)$, $20(46,5 \%)$ responderam corretamente a modalidade CMV (ventilação controlada) e houve um número menor de acertos, 17 (39,5\%), acerca da modalidade SIMV (ventilação mandatória intermitente sincronizada). Os erros são inversamente proporcionais aos acertos; logo, os erros foram maiores em relação ao modo SIMV. Esse elevado número de acertos na Tabela 3 contradiz os erros analisados na Tabela 2, talvez porque a pergunta do questionário tenha sido aberta. 
TABELA3- Distribuição da programação da modalidade a volume e dos alarmes segundo o conhecimento dos enfermeiros. Fortaleza, 2011.

\begin{tabular}{|c|c|c|c|c|c|c|}
\hline MODALIDADE AVOLUME & Correta & $\%$ & Errada & $\%$ & Total & $\%$ \\
\hline $\begin{array}{l}\text { CMV (vertiação mandatória controlada): VC, Fluxo, FR, } \\
\text { PEEP, FiOz, Sensibilidade e Pressão de suporte em OFF }\end{array}$ & 20 & 46,5 & 23 & 53,5 & 43 & 100 \\
\hline $\begin{array}{l}\text { AC (assistida/ cortrolada): VC, Fluxo, FR, PEEP, } \mathrm{FiO}_{2} \text {, } \\
\text { Sensibilidade ligada e Pressão de suporte em OFF }\end{array}$ & 25 & 58,1 & 18 & 41,9 & 43 & 100 \\
\hline $\begin{array}{l}\text { SIMV (vertiação mandatória intermiterte sincronizada): } \\
\text { VC, Fluxo, FR< } \angle=10 \text {, PEEP, FiO2, Pressão de suporte e } \\
\text { alarme de apneia e backup }\end{array}$ & 17 & 39,5 & 26 & 60,5 & 43 & 100 \\
\hline ALARMES & Correta & $\%$ & Errada & $\%$ & Total & $\%$ \\
\hline HIGH PRESSURE LIMIT & 27 & 62,8 & 16 & 37,2 & 43 & 100 \\
\hline LOW PEAK PRESSURE & 19 & 44,2 & 24 & 55,8 & 43 & 100 \\
\hline LOW MINUTE VOLUME & 15 & 34,9 & 28 & 65,1 & 43 & 100 \\
\hline LOW PEEP & 14 & 32,6 & 29 & 67,4 & 43 & 100 \\
\hline
\end{tabular}

Em relação aos sinais dos alarmes, 27 (62,8\%) profissionais souberam relacionar o alarme High Pressure Limit com a problemática, quanto aos demais alarmes a maioria errou. Constata-se que o alarme que o enfermeiro mais observa é alta pressão, que está relacionada à obstrução no circuito por algum motivo, ou seja, o paciente não está sendo ventilado adequadamente.

As perguntas relacionadas às modalidades têm como objetivo que o enfermeiro responda que, ao programar VC, Fluxo, FR 12 - 16 rpm, PEEP, FiO , Sensibilidade (S) ligada e Pressão de suporte (PS) em OFF, o paciente se encontrava na modalidade $A / C$.

Ao programar VC, Fluxo, FR d" 10 rpm, PS, PEEP, $\mathrm{FiO}_{2}$, alarme de apneia e Backup, o enfermeiro deveria perceber que a modalidade do ventilador no qual o paciente se encontra será SIMV. A respiração mandatória é concomitante ao esforço inspiratório do paciente que combina ciclos espontâneos com alguns ciclos mecânicos assistidos, portanto, são sincronizados com o esforço respiratório do paciente. $^{9}$

Quando se programa PEEP e FiO , baixas, Alarme de apneia e Backup, a modalidade do ventilador é Pressão Positiva Contínua nas Vias Aéreas (CPAP). Nessa modalidade, o ventilador permite que o paciente ventile espontaneamente, porém, fornece uma pressão contínua tanto na inspiração quanto na expiração. 0 volume corrente depende do esforço inspiratório do paciente e das condições da mecânica respiratória do pulmão e da parede torácica. ${ }^{3}$ É necessário que o enfermeiro observe se o paciente está suportando bem essa modalidade, se ele não apresenta nenhum desconforto respiratório e se a programação é a ideal.

Ao analisar a participação do enfermeiro na definição dos parâmetros do ventilador mecânico nas UTIs estudadas, os dados mostram que é mínima, ou seja, quase inexiste a atuação do enfermeiro, havendo grande participação do fisioterapeuta, o que comprova que o enfermeiro encontrase distante dessa ação.

É preocupante perceber que, apesar do avanço tecnológico e da capacitação profissional para o cuidado ao paciente crítico, o enfermeiro parece estar dividindo a responsabilidade pela assistência e pelo atendimento das necessidades de oxigenação desses pacientes com outros profissionais, como é o caso da equipe de fisioterapia. ${ }^{14}$

Essa realidade, esse distanciamento do enfermeiro do ventilador, não ocorre somente nos hospitais de Fortaleza-CE. Um estudo realizado em um centro clínico e cirúrgico de terapia intensiva na Zona Sul do Rio de Janeiro identificou que a equipe de enfermagem, talvez por acúmulo e sobrecarga de atividades, aliados à falta de dimensionamento ideal da equipe, tem, cada vez mais, deixado para a equipe de fisioterapia a responsabilidade pela prestação de cuidados junto ao paciente em ventilação mecânica. ${ }^{14}$

Vale ressaltar que, no momento em que 0 enfermeiro não participa ou se torna distante do cuidado relacionado às necessidades dos pacientes em ventilação mecânica, estes se tornam vulneráveis a complicações, como extubações acidentais, infecções, estenose de traqueia, hipoxemia, arritmias respiratórias e cardíacas, tornando, assim, o ambiente de terapia intensiva inseguro para eles. ${ }^{14}$

Cabe salientar que, em relação ao ventilador mecânico, há uma carência que acompanha o enfermeiro desde a graduação até a prática profissional. 0 controle dos alarmes dos ventiladores é uma tarefa importantíssima da enfermagem, no sentido de mantêlos sempre ligados, atentos às possíveis falhas e buscando soluções para estas. ${ }^{15}$

Outro dado importante analisado foi o auxílio durante o processo de extubação do paciente, no qual foi percebido, de acordo com a Tabela 4, que os fisioterapeutas são mais atuantes do que os enfermeiros nesse cuidado, com 58,2\% das respostas. Apenas 11,6\% responderam que o enfermeiro participa e 30,2\% responderam que ambos os profissionais atuam, segundo a Tabela 4. Um estudo mostrou que práticas antes específicas da enfermagem, como montagem do ventilador, definição de parâmetros iniciais, aspiração de vias aéreas, acompanhamento gasométrico, desmame ventilatório, entre outros, estão sendo praticadas por profissionais de outras áreas. ${ }^{16}$ 
TABELA 4 - Distribuição da assistência e intervenções de enfermagem relacionadas à ventilação mecânica. Fortaleza, 2011.

\begin{tabular}{|c|c|c|}
\hline QUEM AUXIUA NA EXTUBAC̄ÃO: & $\mathbf{N}$ & $\%$ \\
\hline Enfermeiro & 5 & 11,6 \\
\hline Fisioterapeuta & 25 & 58,2 \\
\hline Enfermeiro e Fisioterapeuta & 13 & 30,2 \\
\hline Total & 43 & 100,0 \\
\hline QUEMATUA NO PROCESSO DE DESMAME & $\mathbf{N}$ & $\%$ \\
\hline Enfermeiro & 2 & 4,7 \\
\hline Fisioterapeuta & 38 & 88,4 \\
\hline Enfermeiro e Fisioterapeuta & 3 & 6,9 \\
\hline Total & 43 & 100 \\
\hline QUEMASPIRASECREÇOEES TRAQUENS & $\mathbf{N}$ & $\%$ \\
\hline Enfermeiro & 05 & 11,6 \\
\hline Fisioterapeuta & 38 & 88,4 \\
\hline Total & 43 & 100,0 \\
\hline
\end{tabular}

Quanto ao processo de desmame, também se constata que o enfermeiro não é tão atuante, apenas 2 $(4,7 \%)$ informaram que há uma participação do enfermeiro e $3(6,9 \%)$ que dois profissionais participam desse processo. 0 desmame é feito na maioria das vezes pelo fisioterapeuta, com uma par ticipação mais efetiva, correspondendo a um percentual de $88,4 \%$. Quanto à aspiração traqueal, apenas $5(11,6 \%)$ informaram que o enfermeiro realiza a técnica de aspiração traqueal. Ficou evidente que nas UTIs pesquisadas o fisioterapeuta é o profissional que mais atua na VM.

É de grande importância para o estudo as intervenções de enfermagem junto ao paciente em ventilação mecânica, porém, observa-se que as respostas são superficiais. As intervenções mais citadas referem-se a aspiração de secreções pulmonares; umidificação do gás inalado adequadamente; observação do circuito do ventilador (retirada de água, quando necessário, manter e trocar a fixação do TOT/TQT e observação dos alarmes do ventilador, como observado no Quadro 1).

Uma revisão integrativa investigou as intervenções e atividades de enfermagem propostas na literatura para 0 diagnóstico de enfermagem em ventilação espontânea prejudicada. Ela enfatizou a necessidade dos cuidados de enfermagem a pacientes em VM; entretanto, para realizar o cuidado esse profissional precisa de competência técnica. ${ }^{17}$

Esse cuidado é de grande importância, uma vez que a mucosa das vias aéreas sofrerá prejuízo funcional se 0 aquecimento e a umidificação do ar inspirado não forem adequados à combinação de intubação traqueal e ventilação mecânica, com gás não adequadamente umidificado, deslocando a zona de saturação isotérmica para posições mais distais nas vias aéreas; isso resultará em perdas de calor e água da mucosa respiratória e, em casos extremos, causará lesão do epitélio respiratório, com suas implicações clínicas. ${ }^{18}$

\begin{tabular}{ll} 
QUADRO 1- Intervenções de enfermagem relacionadas à VM mais citada pelos enfermeiros \\
\hline INTERVENÇÕES MAS CITADAS & N \\
\hline Aspiração de secreções pulmonares & 19 \\
Umidificação adequada do gás inalado & 16 \\
Observação do circuito do ventilador (retirada de água quando necessário) & 15 \\
Manter e trocar a fixação do TOT/TQT & 14 \\
Observação dos alarmes do ventilador & 9 \\
\hline
\end{tabular}

\section{CONCLUSÃO}

Ao avaliar o conhecimento dos enfermeiros referente à ventilação mecânica nas unidades de terapia intensiva foi constatado que há distanciamento desse profissional em relação a esse suporte, seja por falta de conhecimento ou por esse cuidado ser delegado a um profissional de outra área.

São inúmeras as dificuldades que norteiam a prática de enfermagem, principalmente em UTI, porém, o enfermeiro não pode se distanciar do seu objetivo primordial, que é o cuidar; logo, é essencial que haja um aprofundamento teórico-prático e científico compatível com a complexidade do cuidado desses pacientes e tecnologias direcionadas à assistência deles.

A pesquisa contribui para um aprimoramento da discussão e reflexão acerca da atual assistência de enfermagem prestada a pacientes em uso de ventilador mecânico. Acredita-se na capacidade do enfermeiro, na sua assistência ao paciente em suporte ventilatório, no cuidado de enfermagem visando à prevenção dos possíveis danos e complicações decorrentes da utilização da VM, para alcançar os melhores resultados no atendimento a esses pacientes. 


\section{REFERÊNCIAS}

1. Zuñiga QGP. Ventilação mecânica básica para enfermagem. São Paulo: Atheneu; 2004.

2. Leite MA, Vila VDSC. Dificuldades vivenciadas pela equipe multiprofissional na unidade de terapia intensiva. Rev Latino-Am Enfermagem. 2005 mar/ abr; 13(2): 145-50.

3. Carvalho CRR, Toufen Júnior C, França SA. Ventilação mecânica: princípios, análise gráfica e modalidades ventilatórias. $3^{\circ}$ Consenso Brasileiro de Ventilação Mecânica. J Bras Pneumol. 2007; 33(supl 2): 54-70.

4. Nepomuceno RDM, Silva LD. Pesquisa bibliográfica dos sistemas de vigilância em ventilação mecânica: o estado da arte na enfermagem. Rev Eletr Enferm. 2007; 9(1): 191-99.

5. Sampaio LABN. Ventilação mecânica. In: Calil AM, Paranhos WY. O enfermeiro e as situaç̃̃es de emergência. São Paulo: Atheneu; 2007.

6. Smeltzer SC, Bare BG. Princípios e práticas de reabilitação. Cuidados aos pacientes com distúrbios respiratórios. In: Brunner LS, Suddarth DS. Tratado de enfermagem médico-cirúrgica. $10^{\mathrm{a}}$ ed. Rio de Janeiro: Guanabara Koogan; 2009. p. 1495-560.

7. Conselho Regional de Enfermagem (Ceará). Legislação. Fortaleza: CorenCE; 2008.

8. Backes MTS, Edmann AL, Büscher A, Backes DS. Desenvolvimento e validação de teoria fundamentada em dados sobre o ambiente de unidade de terapia intensiva. Esc Anna Nery. 2011 out/dez; 15(4): 769-75.

9. Leite I. Ventilação mecânica: princípios básicos em enfermagem. 2009. [citado 2011 mar 11]. Disponível em: http://www.joinville.ifsc.edu.br/ josianes/Aulas\%20UTI/ventilacao-mecanica.pdf.

10. Ministério da Saúde(BR). Conselho Nacional de Saúde. Resolução nº 196, de 10 de outubro de 1996. Aprova diretrizes e normas regulamentadoras de pesquisas envolvendo seres humanos. Brasília (DF); 1996.

11. Guerrer FJL, Bianchi ERF. Caracterização do estresse nos enfermeiros de unidades de terapia intensiva. Rev Esc Enferm USP. 2008; 42(2): 35562.

12. Ministério da Saúde(BR). DATASUS. Indicadores e dados básicos [documento on-line]. Brasília(DF); 2006 [citado 2011 mar 11]. Disponível em: http:/tabnet.datasus.gov.br/cgi/idb2004/matriz.htm.

13. Preto VA, Pedrão ப. 0 estresse entre enfermeiros que atuam em unidade de terapia intensiva. Rev Esc Enferm USP. 2009; 43(4): 841-48. 14. Molinaro LC. A enfermagem e a avaliação dos parâmetros oxihemodinâmicos diante da aspiração traqueal de pacientes com ventilação mecânica [dissertação]. Rio de Janeiro: Escola de Enfermagem Anna Nery, Universidade Federal do Rio de Janeiro; 2009 [citado 2011 mar 10]. Disponível em: http://teses2.ufrj.br/51/dissert/ EEAN M LauraCristinaMolinaro.pdf.

15. Bajay HM, Furcolin MIR, Rogante M. Assistência ventilatória mecânica. São Paulo: Ed EPv;1991. p. 27-94.
16. Barreiro Filho RD, Santiago LC, Silva RFA, Cunha KCS. 0 paciente submetido à ventilação mecânica e o cuidado de enfermagem que emerge da prática assistencial. In: Anais do $61^{\circ}$ Congresso Brasileiro de Enfermagem 2009, Fortaleza. [citado 2010 ago 20]. Disponível em: www.abeneventos.com.br/anais_61cben/files/01508.pdf.

17. Santos VFR, Figueiredo AEPL. Intervenção e atividades propostas para o diagnóstico de enfermagem ventilação espontânea prejudicada. Acta Paul Enferm. 2010; 23(6): 824-30.

18. Castro Júnior J. Avaliação dos níveis de umidificação e aquecimento durante a ventilação artificial com a estação de trabalho de anestesia Primus da Dräger com baixo fluxo de gases frescos e permutador de calor e umidade [tese]. Botucatu: Universidade Estadual Paulista; 2009 [citado 2011 mar 10]. Disponível em: http://www.athena.biblioteca.unesp.br/exlibris/bd/ bbo/33004064076P6/2009/castrojunior_j_dr_botfm.pdf. 